EUROPEAN ORGANIZATION FOR NUCLEAR RESEARCH

CERN - AB Department

CERN-AB-2005-033

\title{
A NEW VERSION OF SIXTRACK WITH COLLIMATION AND APERTURE INTERFACE
}

\author{
R. Assmann, S. Redaelli, G. Robert-Demolaize, F. Schmidt \\ CERN, Geneva, Switzerland
}

\begin{abstract}
Simulations of collimation and beam cleaning were so far often performed with simplified computer models. However, the increase in available CPU power has opened the possibility for far more realistic simulations. For large accelerators like LHC it is now possible to track millions of particles, element by element over hundreds of turns. The well established SixTrack code treats the full six-dimensional phase space and considers the non-linear magnet components up to very high order. This code is being used for all LHC tracking simulations and has well developed linear and non-linear error models. SixTrack was extended for tracking of large ensembles of halo particles, taking into account halo interaction with arbitrarily placed collimators. An interface to a program for aperture analysis allows obtaining beam loss maps in the machine aperture. A standardized and portable SixTrack version is now available, providing all functionality of the old SixTrack, as well as the newly added support for halo tracking, collimation and aperture loss maps.
\end{abstract}

Presented at

PAC 2005, Knoxville, USA,

May 16-20, 2005

Geneva, Switzerland

June 2005 


\title{
A NEW VERSION OF SIXTRACK WITH COLLIMATION AND APERTURE INTERFACE
}

\author{
G. Robert-Demolaize, R. Assmann, S. Redaelli, F. Schmidt, CERN, Geneva, Switzerland
}

\section{Abstract}

Simulations of collimation and beam cleaning were so far often performed with simplified computer models. However, the increase in available CPU power has opened the possibility for far more realistic simulations. For large accelerators like LHC it is now possible to track millions of particles, element by element over hundreds of turns.

The well established SixTrack code treats the full sixdimensional phase space and considers the non-linear magnet components up to very high order. This code is being used for all LHC tracking simulations and has well developed linear and non-linear error models. SixTrack was extended for tracking of large ensembles of halo particles, taking into account halo interaction with arbitrarily placed collimators.

An interface to a program for aperture analysis allows obtaining beam loss maps in the machine aperture. A standardized and portable SixTrack version is now available, providing all functionality of the old SixTrack, as well as the newly added support for halo tracking, collimation and aperture loss maps.

\section{INTRODUCTION}

SixTrack [1] is one of the most speed optimized computing code for tracking particles in modern highenergy accelerators. It is based on an element-by-element tracking, using transfer matrices to describe the effect of each lattice element on the beam using a 6-dimensional thin lens formalism [2].

The SixTrack program is the standard tracking tool for the LHC dynamical aperture studies. It takes into account all relevant imperfections, linear and non-linear fields, beam-beam kicks, and other errors for the LHC. It performs fully chromatic and coupled tracking, allowing the treatment of time-dependent field errors and the inclusion of the LHC aperture.

The very first studies for design purpose on the LHC collimation system were done using the K2 scattering procedure and linear transfer matrices (obtained from Twiss functions calculated with MAD). K2 was developed in the 1990's for studies of LHC collimation [3]. After the end of 2000 the K2 routines were included into the COLLTRACK program $[4,5,6]$ which has the following main features:

- Proton scattering in various collimator materials, including single-diffractive scattering.

- Various halo and diffusion models.

- Tracking of large particle ensembles $\left(\sim 10^{6}\right)$ over hundreds of turns.
- Multiple imperfections on the beam and the collimator properties (setting errors, tilts, orbit, beta beat, ...)

The accuracy of the K2 scattering routines was carefully assessed. Comparisons with other commonly used codes [7] showed discrepancies in scattering angles of up to a factor three for large angles. This uncertainty in scattering angles translates into a $30 \%$ change in predicted cleaning inefficiency which is quite reasonable.

COLLTRACK was used extensively for the design of the LHC collimation system, comparing different options in terms of cleaning efficiency. Uncertainties in absolute predictions are induced by its simplified tracking.

For an improved accuracy in cleaning studies it was decided to merge COLLTRACK, SixTrack and an LHC aperture model into a new complete tracking tool.

\section{NEW FEATURES OF SIXTRACK/COLLTRACK}

The SixTrack and the COLLTRACK/K2 source codes were merged with the following goals:

- Modification of SixTrack such as to allow for tracking of large particle ensembles $\left(10^{6}\right.$ protons).

- Implementation of all features of COLLTRACK/K2.

- Implementation of an LHC aperture model with analysis of loss locations for all tracked protons.

- Interface to a computing cluster for a parallelization of computing jobs.

The work on SixTrack started in 2002 with first results presented in [7]. The effort was reinforced in 2004, resulting in the availability of a complete and verified SixTrack/COLLTRACK code.

Considering the number of elements to be treated by the new routines ( 71 collimators per beam for a full system study), it was decided to separate the new input parameters into two classes: beam physics parameters and mechanical parameters, the latest being stored into a separate database file. More information on the changes brought to the SixTrack input can be found at [8].

In order to maintain a good computing performance, the halo tracked in the simulations consists of an annulus in phase space with physical amplitude adjusted so as to impact on the corresponding primary collimator of the betatron cleaning insertion, either the horizontal, vertical or one of the two skew ones (depending on the studied case). Simulations taking into account different diffusion speeds have been done to determine the average impact parameter on the primary collimators, which is used to calculate the normalized amplitude of the annulus [9]. 


\section{EXAMPLE OF SIMULATION RESULTS}

\section{Cleaning Inefficiency Curves}

SixTrack with collimation routines is now able to perform complete tracking of particles through the LHC and its collimation system. For each considered collimator in the sequence of lattice elements, the collimation routines check whether there is a hit and/or absorption. This process is repeated for every particle tracked at every turn. At the end of each turn, the surviving particles are counted in bins that correspond to their normalized amplitude around the closed orbit. A proton is counted if its amplitude is above the lower limit of the specified bin ("integrated inefficiency curve"). Each proton is only counted once in each bin.

The cleaning inefficiency $\eta_{c}$ of the system is defined at a given amplitude $A_{i}$ as the ratio between the number of particles $N_{p}$ with an amplitude above $A_{i}$ and the total number of particles absorbed in the collimation system $N_{a b s}$ :

$$
\eta_{c}\left(A_{i}\right)=\frac{N_{p}\left(A>A_{i}\right)}{N_{a b s}}
$$

A curve like the one in Fig. 1 can then be obtained:

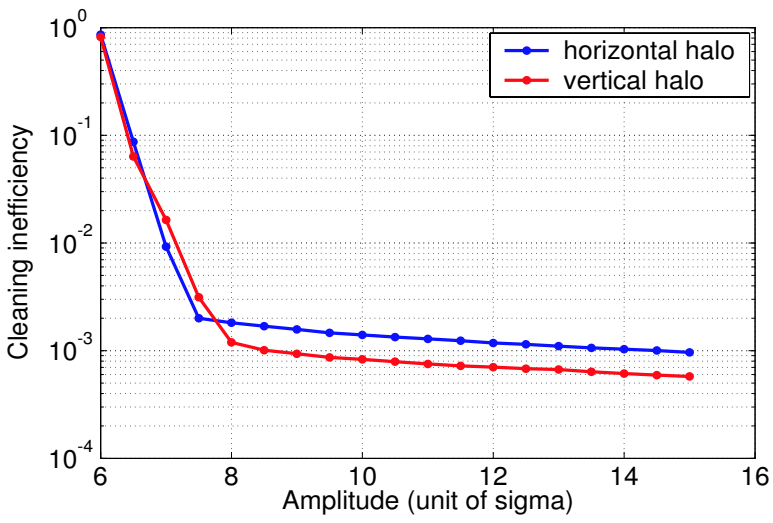

Figure 1: Inefficiency curve for the LHC considering betatron cleaning elements only; case of a vertical halo (red curve) and a horizontal halo (blue curve) tracked at collision optics $(7 \mathrm{TeV})$.

\section{Aperture Program}

The new tracking code can record the trajectories of secondary and tertiary halo particles all along the accelerator. The particle coordinates are recorded at the locations of each magnetic element and are used as an input to an aperture analysis program [10], which is executed immediately after the tracking process.

This aperture program interpolates the saved trajectories along the ring and finds the location where a given trajectory intercepts the LHC aperture within a 10 $\mathrm{cm}$ resolution (which implies interpolation over 270000 points for the LHC lattice). An example of a trajectory of a halo particle hitting the aperture is shown in Fig. 2.

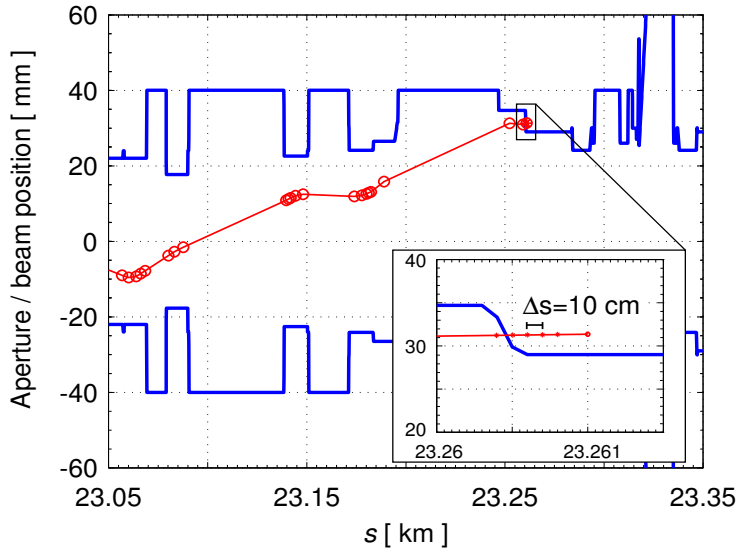

Figure 2: Example of a halo particle (red line) that hits the LHC aperture (blue line) [10].

The aperture model relies on a $2 \mathrm{D}$ treatment of the aperture types of the LHC elements. Linear interpolations between consecutive aperture definitions are applied to cover the full ring.

The new computing package allows obtaining precise maps of losses location along the whole LHC ring, so as to see where the critical regions are, i.e. the locations of possible quenches. By counting the number of particles $N_{\text {loss }}$ lost within $10 \mathrm{~cm}$ long bins, one can define the socalled local cleaning inefficiency as:

$$
\tilde{\eta}_{c}=\frac{1}{\Delta s} \frac{N_{l o s s}}{N_{a b s}}
$$

with $N_{a b s}$ defined as in Eq. 1 and $\Delta s=10 \mathrm{~cm}$. Once obtained, this value is then compared with the quench limit value as defined in [11] for the different LHC optics.

Fig. 3 and 4 show examples of such loss maps, obtained for the same halo tracked and the same optics case, but considering different parts of the LHC collimation system.
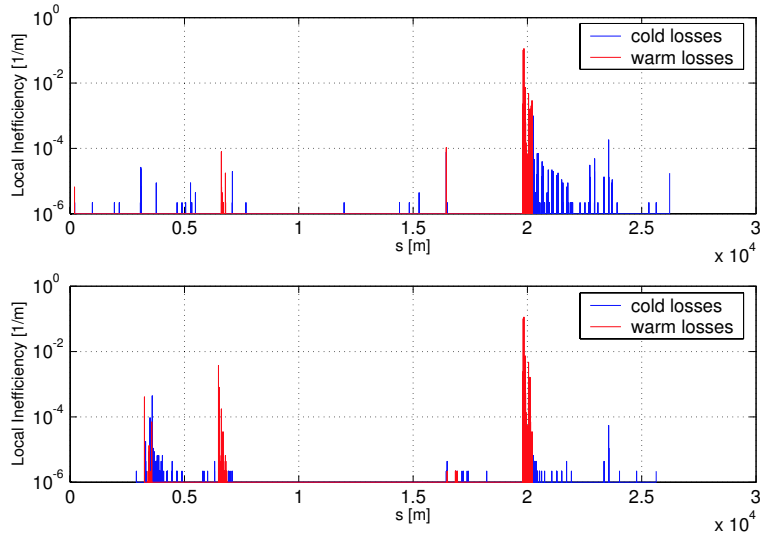

Figure 3: Example of protons losses in the LHC aperture along the LHC ring, for a vertical halo tracked at injection optics; Top: Only betatron cleaning (IR7) is considered; Bottom: The full LHC collimation system is considered. 

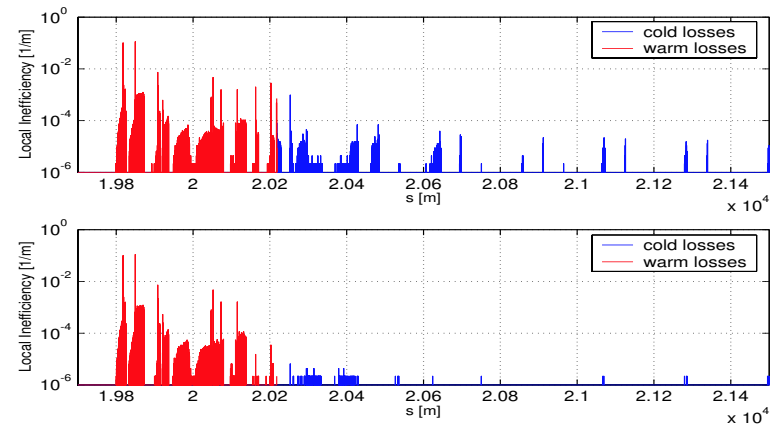

Figure 4: Example of protons losses in the LHC aperture along the IR7 region, for a vertical halo tracked at injection optics; Top: Only betatron cleaning (IR7) is considered; Bottom: The full LHC collimation system is considered.

It can be seen in Fig. 3 and 4 that the complete collimation system (bottom plot) has a significantly better performance than the betatron cleaning system with primary and secondary collimators alone: there is a notable reduction of the loss spikes in the arc downstream of the IR7 insertion region, where the betatron cleaning is located. At the same time, additional losses are observed in IR2, where the TDI injection protection element is intercepting the secondary halo at its nominal setting.

These observations illustrate the powerful insights into the LHC cleaning performance. Understanding the complicated cleaning processes and multi-turn losses in the LHC must rely on powerful computing tools, as they are described in this paper.

\section{Loss Patterns along Collimator Jaws}

When an absorption (inelastic interaction) occurs in a given jaw, the code saves the coordinates $\left(x, x, y, y^{\prime}\right)$ of the particle and also its longitudinal position along the jaw and its energy change. One can then see both the transverse and the longitudinal (Fig. 5) distribution of absorptions for any collimation system element. These data are used as an input for detailed showering codes that describe and further track the particle showers from the inelastic interaction onwards [12].

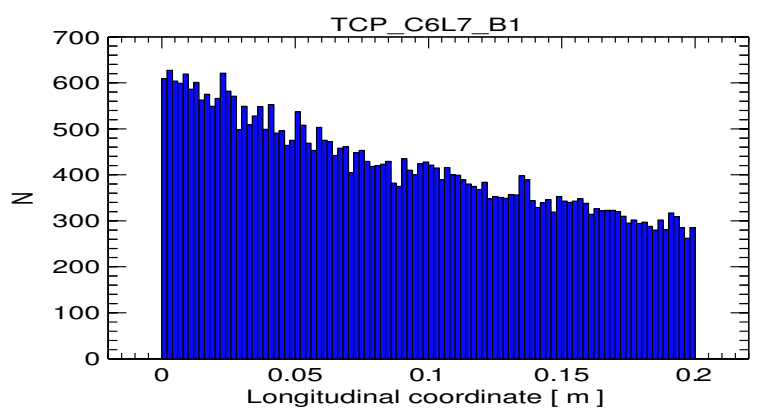

Figure 5: Longitudinal distribution of the horizontal halo protons absorbed at element TCLP.C6L7.B1, first horizontal primary collimator of LHC betatron cleaning insertion.

\section{CONCLUSION}

It is now possible to perform detailed simulations of the LHC cleaning processes and multi-turn loss patterns around the LHC ring. The simulations can include all collimators, diluters and absorbers that are foreseen. The use of state-of-the-art computer codes allows to perform such studies with an accuracy hardly achieved before (tracking of millions of halo particles over hundreds of turns with proton-matter interactions). Proton loss maps in the mechanical aperture are generated with a $10 \mathrm{~cm}$ resolution. This makes it possible to perform advanced studies for quenches of super-conducting magnets along with the analysis of the deposited energy in any given jaw. Only ideal machine cases have been studied so far with the full model, but the simulation of various imperfections and accident cases are foreseen in the near future.

\section{ACKNOWLEDGMENTS}

The authors would like to thank J.B. Jeanneret and D. Kaltchev for their help in setting up the collimation models. Many thanks to E. McIntosh for his help in the code development, and to O. Bruening and T. Risselada for their help in setting up the optics files.

\section{REFERENCES}

[1] F. Schmidt: "SixTrack, User's Reference Manual", CERN SL/94-56 (AP), 1994.

[2] G. Ripken, F. Schmidt: "A symplectic six-dimensional thin-lens formalism for tracking”, CERN-SL-95-12, 1995.

[3] T. Trenkler, J.B. Jeanneret: "K2, A software package evaluating collimation systems in circular colliders (manual)", CERN SL/94-105 (AP), 1994.

[4] R. Assmann, J.B. Jeanneret, D. Kaltchev: "Status of Robustness Studies for the LHC Collimation", APAC 2001.

[5] R. Assmann, J.B. Jeanneret, D. Kaltchev: "Efficiency for the Imperfect LHC Collimation System", EPAC 2002.

[6] R. Assmann: "Collimators and Cleaning, Could this Limit the LHC Performance?”, Proc. Chamonix 2003.

[7] R. Assmann et al.: "Tools for Predicting Cleaning Efficiency in the LHC”, LHC Project Report 639, 2003.

[8] http://lhc-collimation-project.web.cern.ch/lhccollimation-project/code-tracking.htm

[9] R. Assmann, F. Schmidt, F. Zimmermann: "Equilibrium Beam Distribution and Halo in the LHC", EPAC 2002.

[10] S. Redaelli: "LHC Aperture and Commissioning of the Collimation System”, Proc. Chamonix 2005.

[11] J.B. Jeanneret, D. Leroy, L. Oberli and T. Trenckler: "Quench levels and transient beam losses in the LHC magnets", CERN-LHC-PROJECT-REPORT-44 (1996).

[12] M. Santana, "Energy Deposition Studies for the Betatron Cleaning Insertion", these proceedings. 